\title{
A Study of Tongue and Pulse Diagnosis in Traditional Korean Medicine for Stroke Patients Based on Quantification Theory Type II
}

\author{
Mi Mi Ko, ${ }^{1}$ Tae-Yong Park, ${ }^{1}$ Ju Ah Lee, ${ }^{1}$ Byoung-Kab Kang, \\ Jungsup Lee, ${ }^{2}$ and Myeong Soo Lee ${ }^{1}$ \\ ${ }^{1}$ Medical Research Division, Korea Institute of Oriental Medicine, 1672 Yuseongdae-ro, Yuseong-gu, Daejeon 305-811, Republic of Korea \\ ${ }^{2}$ Department of Oriental Rehabilitation Medicine, Korean National Rehabilitation Center, 58 Samgaksan-ro, Gangbuk-gu, \\ Seoul 142-884, Republic of Korea \\ Correspondence should be addressed to Ju Ah Lee; motoong@kiom.re.kr
}

Received 23 November 2012; Revised 18 March 2013; Accepted 19 March 2013

Academic Editor: Adair Roberto Soares Santos

Copyright (c) $2013 \mathrm{Mi}$ Mi Ko et al. This is an open access article distributed under the Creative Commons Attribution License, which permits unrestricted use, distribution, and reproduction in any medium, provided the original work is properly cited.

\begin{abstract}
In traditional Korean medicine (TKM), pattern identification (PI) diagnosis is important for treating diseases. The aim of this study was to comprehensively investigate the relationship between the PI type and tongue diagnosis or pulse diagnosis variables. The study included 1,879 stroke patients who were admitted to 12 oriental medical university hospitals from June 2006 through March 2009. The status of the pulse and tongue was examined in each patient. Additionally, to investigate relatively important indicators related to specialist PI, the quantification theory type II analysis was performed regarding the PI type. In the first axis quantification of the external criteria, the Qi-deficiency and the Yin-deficiency patterns were located in the negative direction, while the dampnessphlegm (DP) and fire-heat patterns were located in the positive direction. The explanatory variable with the greatest impact on the assessment was a fine pulse. In the second axis quantification, the external criteria were divided into either the DP or non-DP patterns. The slippery pulse exhibited the greatest effect on the division. This study attempted to build a model using a statistical method to objectively quantify PI and various indicators that constitute the unique diagnosis system of TKM. These results should assist the development of future diagnostic standards in stroke PI.
\end{abstract}

\section{Introduction}

Traditional Korean medicine (TKM) uses a unique diagnostic system of pattern identification (PI) based on the indicated reactions of the body to disease [1]. TKM uses the four methods of diagnosis, which include diagnosis by observation, diagnosis by hearing and smelling, diagnosis by interrogation, and diagnosis by palpation $[1,2]$. One of the typical methods of diagnosis by observation is tongue diagnosis, which is the evaluation of a disease by observing the tongue. This method is actively used to examine the causes, properties, and affected areas of a disease and to determine the prognosis of a disease by observing changes in a patient's tongue characteristics and tongue coating. The most representative method of diagnosis involves palpation and is known as pulse diagnosis. Pulse diagnosis is an examination technique in which the doctor directly palpates the pulses on both wrists of a patient to evaluate the properties and condition of the pulses. A patient's condition and disease are diagnosed according to the palpation of the pulse, a treatment plan is chosen, and the effectiveness of the selected treatment is determined by comparing the pulses before and after the treatment.

The definition of "pattern" in TKM refers to the overview of each step in the course of a disease and consists of a combination of correlations between several symptoms and signs [3]. Previous reports have described the PI process for differentiating stroke victims with four TKM types: the fireheat pattern, dampness-phlegm pattern, Yin-deficiency pattern, and Qi-deficiency pattern [4-6]. However, the meaning of a pattern is often ambiguous in clinical practice, and it is difficult to objectively arrange or accumulate the clinical 
data regarding these patterns or perform a systematic analysis of the results. TKM emphasizes PI as an expedient for treatment by specifying the nature of a disease, but collecting quantitative data for the assessments is difficult because the assessments rely on the patient's subjective descriptions and the doctor's subjective judgments.

The quantification theory applied in this study follows the theory of the quantification of qualitative data developed by Hayashi in Japan after 1950 [7]. This quantification theory consists of four types classified as I, II, III, and IV. The quantification of the type II analysis used in this study uses a canonical correlation analysis or a canonical discriminant analysis when both the external criteria and explanatory variables are qualitative $[7,8]$. This quantification method examines the correlation among the categories of dependent variables and the categories of covariates, and it is very useful in the medical research field for the estimation, diagnosis, prognosis, and evaluation of epidemiological factors [9]. Previous studies have used this quantification method in TKM [10-12]. Recently, we conducted a quantification theory method to investigate the relationship between PI types and its properties for using stroke patients from TKM hospitals [12].

Hayashi's quantification type II analysis was performed in the current study using variable data from tongue diagnosis and pulse diagnosis. The data were collected from case report forms (CRFs) with a focus on PI for stroke patients at $12 \mathrm{TKM}$ hospitals throughout the nation [5]. Using this analysis, we aimed to comprehensively investigate the correlations among the PI diagnosed by a specialist, tongue diagnosis, and pulse diagnosis.

\section{Methods}

2.1. Study Subjects. As part of the research on the standardization of oriental medicine stroke PI, the Korea Institute of Oriental Medicine (KIOM) collected stroke data for stroke PI from June 2006 to March 2009 [4-6]. The data were obtained from 12 TKM hospitals and oriental medical universities throughout the nation (Figure 1). The eligibility inclusion criteria included enrolled stroke patients within 30 days of the onset of symptoms as confirmed by imaging diagnosis, such as computerized tomography (CT) or magnetic resonance imaging (MRI). The exclusion criteria included traumatic stroke patients, such as patients with subarachnoid, subdural, or epidural hemorrhages. Two clinical specialists who were well trained in the standard operation procedures (SOPs) simultaneously examined the patterns of identification for a patient, and the symptoms present within 24 hours of the time of examination were used as the basis for analysis. The specialists had at least 3 years of clinical experience with stroke patients after finishing regular college education for TKM for 6 years. Based on the symptoms described by the patients, the doctor's objective judgments were recorded. Among the data collected in this manner, 1,879 data entries with PI by the 2 specialists were used for the analysis. All the patients provided written informed consent under procedures approved by the institutional review boards (IRB).
2.2. Data Processing and Analysis. The chi-square test and Fisher's exact test were used for the analysis of discrete variables. The Kolmogorov-Smirnov method was used to test the normality of the continuous variables, which were analyzed using one-way ANOVA (as a parametric method) and the Kruskal-Wallis test (as a nonparametric method). The examination parameters were extracted from CRFs for the standardization of stroke diagnosis developed by an expert committee organized by the KIOM [4-6]. Each patient received an examination of the status of the tongue and pulse, tongue color (pale, pale-red, red, or bluish purple), fur color (white fur or yellow fur), fur quality (thick fur or dry fur), special tongue appearance (teeth marked, enlarged, mirror, or spotted), pulse location (floating or sunken), pulse rate (slow or rapid), pulse force (strong or weak), and pulse shape (fine, slippery, rough, or surging). The analysis was performed by converting the index measurements obtained using the 3 -point scale in which $3=$ very much, $2=$ much, and $1=$ not much into a 2 -point scale in which $1=$ yes and $0=$ no. The information of the measurement variables is shown in Supplementary Table 1 (see Supplementary Material available online at http://dx.doi.org/10.1155/2013/508918). The PI data assessed in this study included the results of the 4 PIs, which were measured as fire-heat (FH), dampnessphlegm (DP), Qi-deficiency (QD), and Yin-deficiency (YD) patterns. These assessments were given individually without discussions among the specialists. A total of 1,879 stroke patients received a PI assessment with the same opinions by specialists with the following distribution: $\mathrm{FH}$ pattern $(n=$ $567)$, DP pattern $(n=664)$, YD pattern $(n=279)$, and QD pattern $(n=369)$ (Figure 1$)$.

The quantification type II theory, which is a multivariate analysis, is suitable when predicting qualitative values as the external criteria variable based on the information concerning the qualitative explanatory variables of each subject $[7,8]$. Most of the variables in this study are categorical, and the quantification type II theory based on canonical correlation analysis (CCA) was applied to identify the relationship between two sets of multidimensional variables including the 4 types of PI and the tongue/pulse diagnosis variables. Concurrence between the 2 specialists served as the external criteria, and the tongue diagnosis and pulse diagnosis variables served as the explanatory variables (see Supplementary Table 2).

The CCA was conducted first for the quantification type II theory. Raw canonical coefficients that were used for the quantification type II theory were derived from the CCA. After finding the raw canonical coefficients, the quantification value was calculated. We calculated the centering value $\left(c_{p}\right)(1)$ based on the raw canonical coefficients and obtained the categorical score $\left(s_{p q}\right)$ using a simple formula $(2)$ as

$$
c_{p}=\frac{\sum_{q=1}^{n_{p}} a_{p q} \times x_{p q}}{\sum_{q=1}^{n_{p}} x_{p q}},
$$

where $x_{p q}$ is the frequency of level $q$ of the $p$ th category variable, $a_{p q}$ is the associated with level $q$ of each $p$ th category variable, and $n_{p}$ is the total number of $p$ th category variables. 


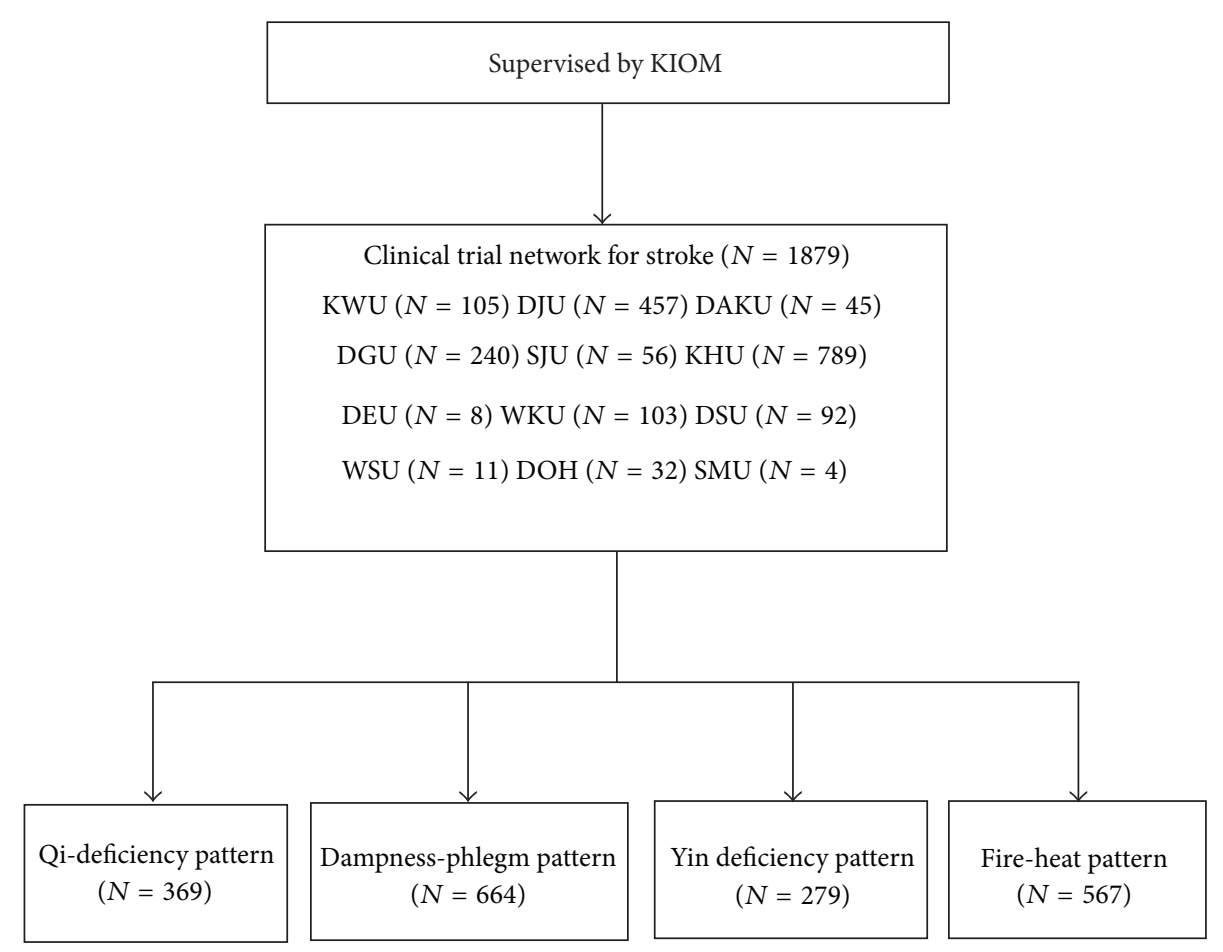

FIGURE 1: Flow chart showing patient enrollment in study. KIOM, Korean Institute of Oriental Medicine; KWU, Kyung Won Oriental Medical Hospital; DJU, Dae Jeon Oriental Medical Hospital; DAKU, Dae Gu Hanny University Medical Center; DGU, Dong Guk International Hospital; SJU, Sang Ji Oriental Medical Hospital; KHU, Kyung Hee Oriental Medical Hospital; DEU, Dong Eui Oriental Medical Hospital; WKU, Won Kwang Oriental Medical Hospital; DSU, Dong Sin Oriental Medical Hospital; WSU, Woo Suk Oriental Medical Hospital; DOH, Dong Seo Oriental Medical Hospital; SMU, Se Myeong Oriental Medical Hospital; QD, Qi-deficiency pattern; DP, dampness-phlegm pattern; YD, Yin-deficiency pattern; FH, fire-heat pattern.

Consider the following:

$$
s_{p q}=a_{p q}-c_{p},
$$

where $s_{p q}$ is the categorical score (quantification value) of level $q$ of the $p$ th category variable.

The quantification range represents the difference between the maximum quantification value and the minimum quantification value, and it is used as an indicator with the partial correlation coefficient to show the importance of each explained variance.

SAS (Version 9.1.3, SAS Institute Inc., Cary, NC, USA) was used for the statistical analysis.

\section{Results}

The general characteristics of the study subjects are shown in Table 1.

Throughout the CCA for the quantification type II analysis, the canonical correlation coefficient for $Y 1$ and $X 1$ in the first axis was 0.585 , and the canonical correlation coefficient of $Y 2$ and $X 2$ in the second axis was 0.555 (Table 2). The third axis was not considered because the canonical correlation coefficient in the third axis was relatively small compared with the other axes. The first two axes explain $91.54 \%$ of the total data (first axis: $49.29 \%$, second axis: $42.25 \%$ ).
The first axis quantification for the external criteria consisted of assessments of (-) QD, YD, DP, and FH (+) axes. The QD and the YD were located at $(-)$ quantification values of -1.5990 and -0.6004 , respectively, while the DP and the $\mathrm{FH}$ values were located at (+) 0.1103 and 1.2069 , respectively (Table 3). Based on the quantification range, the explained variance with the highest correlation corresponds with the fine pulse state. The assessments of fine pulse $(-0.3731)$ were related to the $\mathrm{QD}$ and the $\mathrm{YD}$, whereas the assessments without fine pulse (0.0958) were related to the DP and the $\mathrm{FH}$ patterns. Pale tongue (a type of tongue diagnosis) also demonstrated considerable significance as an explanatory factor; the demonstrations of pale tongue $(-0.1223)$ was predicted the diagnoses of QD and YD. Furthermore, yellow fur (a type of tongue diagnosis) followed by weak pulse (a type of pulse states) and surging pulse (a type of pulse state) was shown to be significant explanatory factors (Tables 4 and 5).

Regarding the external criteria, the second axis quantification included the contrast between the (-) DP and non-DP (+) assessments (Table 3 ). Based on the quantification range, the factor with the highest correlation was the slippery pulse. The assessments of slippery pulse (0.0582) were related to the DP quality. Spotted tongue (a type of tongue diagnosis) also demonstrated considerable significance as an explanatory factor; cases with spotted tongue (0.1710) were correlated with 
TABLE 1: Demographic parameters of study subjects.

\begin{tabular}{|c|c|c|c|c|c|}
\hline Characteristics & Fire-heat & Dampness-phlegm & Qi deficiency & Yin deficiency & $P$ \\
\hline$N$ & 567 & 664 & 369 & 279 & \\
\hline $\operatorname{Sex}(M / F)$ & $425 / 142$ & $306 / 358$ & $121 / 248$ & $128 / 151$ & $* *$ \\
\hline Age $($ Mean \pm SD $)$ & $65.50 \pm 11.82$ & $66.41 \pm 11.13$ & $67.31 \pm 11.89$ & $69.54 \pm 11.84$ & $* *$ \\
\hline Weight $(\mathrm{kg})($ Mean \pm SD $)$ & $65.09 \pm 10.55$ & $63.77 \pm 10.59$ & $56.63 \pm 9.21$ & $57.42 \pm 11.10$ & $* *$ \\
\hline Height $(\mathrm{cm})($ Mean $\pm \mathrm{SD})$ & $164.07 \pm 8.42$ & $160.37 \pm 8.71$ & $157.51 \pm 8.11$ & $158.54 \pm 12.14$ & $* *$ \\
\hline $\mathrm{BMI}($ Mean $\pm \mathrm{SD})$ & $24.23 \pm 2.93$ & $24.74 \pm 3.20$ & $22.78 \pm 2.97$ & $22.79 \pm 4.09$ & $* *$ \\
\hline WHR $($ Mean \pm SD $)$ & $0.95 \pm 0.09$ & $0.94 \pm 0.08$ & $0.92 \pm 0.10$ & $0.93 \pm 0.13$ & $* *$ \\
\hline $\mathrm{WC}(\mathrm{cm})($ Mean $\pm \mathrm{SD})$ & $88.62 \pm 9.36$ & $88.77 \pm 9.57$ & $84.46 \pm 8.97$ & $83.00 \pm 9.30$ & $* *$ \\
\hline $\mathrm{HC}(\mathrm{cm})($ Mean $\pm \mathrm{SD})$ & $94.10 \pm 9.21$ & $94.27 \pm 9.28$ & $91.67 \pm 9.28$ & $90.21 \pm 10.62$ & $* *$ \\
\hline \multicolumn{6}{|l|}{ TOAST classification } \\
\hline LAA & 153 & 138 & 62 & 66 & $*$ \\
\hline $\mathrm{CE}$ & 40 & 31 & 23 & 19 & \\
\hline SVO & 271 & 402 & 215 & 138 & \\
\hline SOE & 5 & 11 & 7 & 8 & \\
\hline SUE & 23 & 29 & 14 & 10 & \\
\hline Hypertension (yes, no) & $332 / 233$ & $419 / 243$ & $217 / 147$ & $161 / 118$ & NS \\
\hline Hyperlipidemia (yes, no) & $77 / 478$ & $86 / 572$ & $44 / 320$ & $24 / 249$ & NS \\
\hline DM (yes, no) & $144 / 417$ & $192 / 469$ & $97 / 268$ & $66 / 211$ & NS \\
\hline Smoking (none/stop/active) & $220 / 127 / 220$ & $387 / 88 / 186$ & $239 / 44 / 85$ & $154 / 42 / 83$ & $* *$ \\
\hline Drinking (none/stop/active) & $230 / 74 / 263$ & $389 / 60 / 212$ & $223 / 36 / 109$ & $151 / 29 / 99$ & $* *$ \\
\hline
\end{tabular}

BMI: body mass index. WHR: waist hip ratio. WC: waist circumference. HC: hip circumference. TOAST: trial of ORG 10172 in acute stroke treatment. LAA: large-artery atherosclerosis. CE: cardioembolism. SVO: small-vessel occlusion. SOE: stroke of other etiology. SUE: stroke of undetermined etiology. DM: diabetes mellitus. NS: not significant. ${ }^{*} P<.0001 .{ }^{*} P<.01$.

TABLE 2: Results of the canonical correlation analysis.

\begin{tabular}{lcccccc}
\hline Variate number & Canonical correlation & Approximate standard error & Eigenvalue & Proportion & Cumulative & $P$ \\
\hline 1 & 0.5848 & 0.0152 & 0.5193 & 0.4929 & 0.4929 & $* *$ \\
2 & 0.5550 & 0.0159 & 0.4452 & 0.4225 & 0.9154 & $* *$ \\
3 & 0.2861 & 0.0212 & 0.0891 & 0.0846 & 1 \\
\hline$* * * 0001$ & & & &
\end{tabular}

TABLE 3: Calculated result by Hayashi's quantification method type 2: external criterion.

\begin{tabular}{lcccc}
\hline External criterion & \multicolumn{2}{c}{ First axis } & \multicolumn{2}{c}{ Second axis } \\
& $\begin{array}{l}\text { Category } \\
\text { scores }\end{array}$ & Range & $\begin{array}{c}\text { Category } \\
\text { scores }\end{array}$ & Range \\
\hline Fire-heat & 1.2069 & 2.8059 & -0.7094 & 2.5485 \\
Dampness-phlegm & 0.1103 & & 1.3007 & \\
Qi deficiency & -1.5990 & & -0.3071 & \\
Yin deficiency & -0.6004 & & -1.2478 & \\
\hline
\end{tabular}

the DP readout. Pale tongue (a type of tongue diagnosis) and dry fur were significant explanatory factors (Tables 4 and 5).

Figure 2 shows the quantification plot using the quantification values of the first axis and the second axis for the 4 types of PI as the external properties, and Figure 3 shows the quantification plot using the quantification values of the

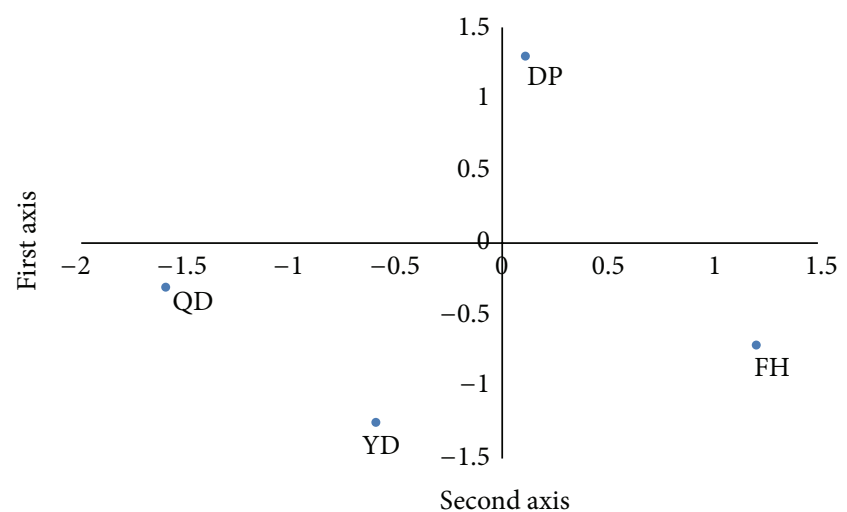

FIGURE 2: Quantification plot of external criterion. FH, fire-heat; DP, dampness-phlegm; QD, Qi-deficiency; YD, Yin-deficiency patterns.

first axis and the second axis for the indicators as explanatory variables. 
TABLE 4: Calculated result by Hayashi's quantification method type 2: tongue indicators.

\begin{tabular}{|c|c|c|c|c|c|c|}
\hline \multirow{2}{*}{ Variable } & \multicolumn{3}{|c|}{ First axis } & \multicolumn{3}{|c|}{ Second axis } \\
\hline & Category scores & Range & Partial correlation $^{\ddagger}$ & Category scores & Range & Partial correlation $^{\ddagger}$ \\
\hline \multicolumn{7}{|l|}{ Pale } \\
\hline $\mathrm{y}$ & -0.1223 & 0.4241 & 0.0871 & 0.1065 & 0.3693 & 0.0741 \\
\hline $\mathrm{n}$ & 0.3018 & & & -0.2628 & & \\
\hline \multicolumn{7}{|l|}{ Palered } \\
\hline $\mathrm{y}$ & -0.0903 & 0.3161 & 0.0657 & 0.0644 & 0.2254 & 0.0457 \\
\hline $\mathrm{n}$ & 0.2258 & & & -0.1610 & & \\
\hline \multicolumn{7}{|l|}{ Red } \\
\hline $\mathrm{y}$ & 0.0019 & 0.0080 & 0.0017 & -0.0288 & 0.1230 & 0.0250 \\
\hline $\mathrm{n}$ & -0.0061 & & & 0.0942 & & \\
\hline \multicolumn{7}{|c|}{ Bluish purple } \\
\hline $\mathrm{y}$ & -0.0790 & 0.0970 & 0.0183 & -0.2676 & 0.3285 & 0.0605 \\
\hline $\mathrm{n}$ & 0.0180 & & & 0.0609 & & \\
\hline \multicolumn{7}{|c|}{ Yellow fur } \\
\hline y & 0.1125 & 0.3863 & 0.1504 & 0.0216 & 0.0741 & 0.0285 \\
\hline $\mathrm{n}$ & -0.2738 & & & -0.0525 & & \\
\hline \multicolumn{7}{|c|}{ White fur } \\
\hline y & 0.0151 & 0.1048 & 0.0460 & 0.0411 & 0.2858 & 0.1215 \\
\hline $\mathrm{n}$ & -0.0897 & & & -0.2447 & & \\
\hline \multicolumn{7}{|l|}{ Thick fur } \\
\hline y & 0.0617 & 0.1604 & 0.0854 & 0.0593 & 0.1542 & 0.0802 \\
\hline $\mathrm{n}$ & -0.0987 & & & -0.0949 & & \\
\hline \multicolumn{7}{|l|}{ Dry fur } \\
\hline y & 0.0085 & 0.0147 & 0.0071 & -0.1706 & 0.2952 & 0.1381 \\
\hline $\mathrm{n}$ & -0.0062 & & & 0.1245 & & \\
\hline \multicolumn{7}{|c|}{ Teeth marked } \\
\hline y & -0.0978 & 0.1753 & 0.0745 & 0.0161 & 0.0289 & 0.0120 \\
\hline $\mathrm{n}$ & 0.0775 & & & -0.0128 & & \\
\hline \multicolumn{7}{|l|}{ Enlarged } \\
\hline $\mathrm{y}$ & -0.0632 & 0.1192 & 0.0519 & 0.0726 & 0.1371 & 0.0582 \\
\hline $\mathrm{n}$ & 0.0561 & & & -0.0645 & & \\
\hline \multicolumn{7}{|l|}{ Spotted } \\
\hline $\mathrm{y}$ & 0.1710 & 0.1812 & 0.0234 & 0.3865 & 0.4097 & 0.0516 \\
\hline $\mathrm{n}$ & -0.0102 & & & -0.0232 & & \\
\hline \multicolumn{7}{|l|}{ Mirror } \\
\hline y & -0.1415 & 0.1691 & 0.0376 & -0.2023 & 0.2416 & 0.0523 \\
\hline $\mathrm{n}$ & 0.0275 & & & 0.0393 & & \\
\hline
\end{tabular}

${ }^{\ddagger}$ Partial correlation coefficient. y and $\mathrm{n}$ denote yes and no, respectively.

\section{Discussion}

For the diagnosis and treatment of stroke, TKM has established a principle of diagnosis based on various patient symptoms using a method known as PI to identify symptoms resulting from a diseased condition or the development of a disease in the human body [1]. The process of PI integrates many symptoms, and tongue diagnosis and pulse diagnosis serve as important disease indicators [2].

As a part of the SOPI-STROKE project, the KIOM recently created the Korean standard PI for stroke (K-SPI) for use in Korea [4-6]. This system consists of 4 types of PI (FH, DP, YD, and QD) and 44 types of indicators [5]. The $\mathrm{FH}$ pattern is characterized by any symptom of heat or fire 
TABLE 5: Calculated result by Hayashi's quantification method type 2: pulse indicators.

\begin{tabular}{|c|c|c|c|c|c|c|}
\hline \multirow{2}{*}{ Variable } & \multicolumn{3}{|c|}{ First axis } & \multicolumn{3}{|c|}{ Second axis } \\
\hline & Category scores & Range & Partial correlation ${ }^{\ddagger}$ & Category scores & Range & Partial correlation ${ }^{\ddagger}$ \\
\hline \multicolumn{7}{|l|}{ Floating } \\
\hline $\mathrm{y}$ & -0.0214 & 0.0697 & 0.0316 & 0.0387 & 0.1259 & 0.0557 \\
\hline $\mathrm{n}$ & 0.0483 & & & -0.0872 & & \\
\hline \multicolumn{7}{|l|}{ Sunken } \\
\hline $\mathrm{y}$ & -0.0480 & 0.1384 & 0.0621 & 0.0804 & 0.2319 & 0.1012 \\
\hline $\mathrm{n}$ & 0.0904 & & & -0.1515 & & \\
\hline \multicolumn{7}{|l|}{ Slow } \\
\hline $\mathrm{y}$ & -0.0092 & 0.0175 & 0.0071 & 0.1103 & 0.2102 & 0.0833 \\
\hline $\mathrm{n}$ & 0.0083 & & & -0.0998 & & \\
\hline \multicolumn{7}{|l|}{ Rapid } \\
\hline y & 0.0625 & 0.2152 & 0.1085 & 0.0003 & 0.0011 & 0.0005 \\
\hline $\mathrm{n}$ & -0.1526 & & & -0.0008 & & \\
\hline \multicolumn{7}{|l|}{ Strong } \\
\hline $\mathrm{y}$ & 0.0501 & 0.1850 & 0.0842 & -0.0236 & 0.0870 & 0.0387 \\
\hline $\mathrm{n}$ & -0.1349 & & & 0.0634 & & \\
\hline \multicolumn{7}{|l|}{ Weak } \\
\hline $\mathrm{y}$ & -0.1347 & 0.3806 & 0.1552 & -0.0439 & 0.1240 & 0.0499 \\
\hline $\mathrm{n}$ & 0.2459 & & & 0.0801 & & \\
\hline \multicolumn{7}{|l|}{ Fine } \\
\hline $\mathrm{y}$ & -0.3731 & 0.4688 & 0.1954 & -0.1815 & 0.2281 & 0.0941 \\
\hline $\mathrm{n}$ & 0.0958 & & & 0.0466 & & \\
\hline \multicolumn{7}{|l|}{ Slippery } \\
\hline $\mathrm{y}$ & 0.0582 & 0.1042 & 0.0573 & 0.3816 & 0.6828 & 0.3447 \\
\hline $\mathrm{n}$ & -0.0460 & & & -0.3012 & & \\
\hline \multicolumn{7}{|l|}{ Rough } \\
\hline $\mathrm{y}$ & -0.0331 & 0.0347 & 0.0088 & 0.0020 & 0.0021 & 0.0005 \\
\hline $\mathrm{n}$ & 0.0016 & & & -0.0001 & & \\
\hline \multicolumn{7}{|l|}{ Surging } \\
\hline $\mathrm{y}$ & 0.3368 & 0.3715 & 0.1253 & -0.2073 & 0.2286 & 0.0756 \\
\hline $\mathrm{n}$ & -0.0347 & & & 0.0213 & & \\
\hline
\end{tabular}

${ }^{\ddagger}$ Partial correlation coefficient. y and $\mathrm{n}$ denote yes and no, respectively.

that is contracted externally or engendered internally. The DP pattern is characterized by impeding Qi movement and its turbidity, heaviness, stickiness, and downward-flowing properties. The QD pattern is characterized by Qi deficiency with diminished internal organ function, which is marked by shortness of breath, lassitude, listlessness, spontaneous sweating, a pale tongue, and a weak pulse. The YD pattern is characterized by Yin deficiency with diminished moistening and the inability to restrain yang, which is usually manifested as fever [4-6].

This study aimed to examine the correlation between stroke PI and tongue diagnosis or pulse diagnosis variables using a quantitative analysis. The quantification theory type II is suitable when predicting the qualitative data as the external criteria variable based on the information concerning the qualitative explanatory variables of each subject. Shin [10] suggested the quantification theory type II method to quantify the knowledge obtained in the process of TKM diagnoses using stroke data but used a small sample size of only 45 stroke patients. No pulse information and few tongue indicators were obtained, which makes it difficult to generalize the results. In 2010, we conducted the quantification theory type II method to investigate the relationship between PI types and properties using 835 stroke patients from TKM hospitals [12]. The pulse status and tongue status were evaluated along with other items (e.g., headaches, dizziness, facial complexion items, skin items, urine items, etc.). However, the discriminative estimation of the YD pattern is relatively low compared with other patterns. In this study, as a result of the first axis quantification of the external criteria, the QD and the YD were located in the negative direction, while the $\mathrm{DP}$ and $\mathrm{FH}$ patterns were located in the positive direction, and the locations were used to differentiate between excess and deficiency. There are many methods for PI diagnosis in TKM, and PI diagnosis according to the eight guiding principles is the basis for all the other methods. It involves the categorization of a patient's condition according to four opposing pairs of principles: interior/exterior, heat/cold, 


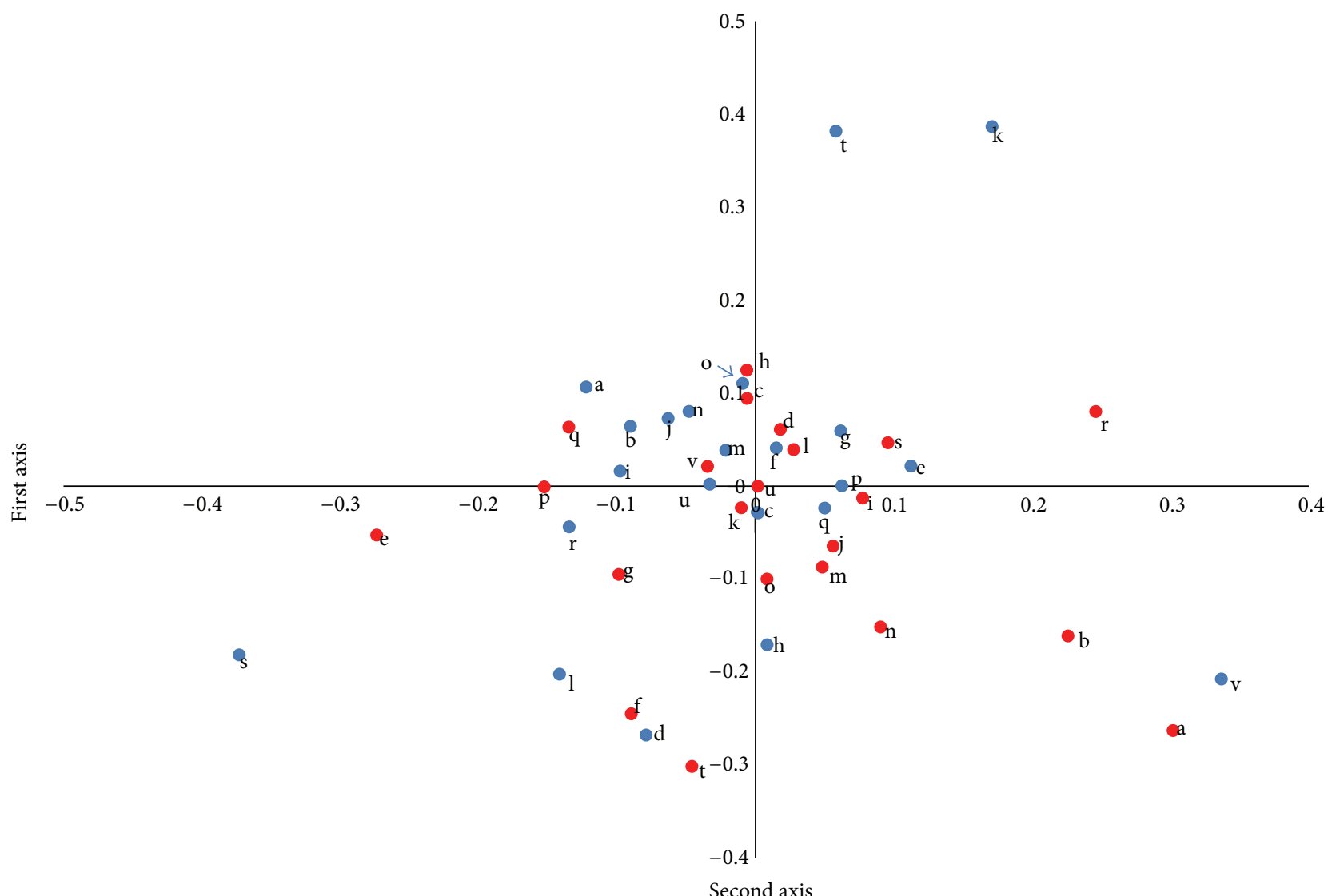

FIGURE 3: Quantification plot of independent variables. a, pale; b, pale red; c, red; d, bluish purple; e, yellow fur; f, white fur; g, thick fur; h, dry fur; i, teeth marked; j, enlarged; k, spotted; l, mirror; m, floating; n, sunken; o, slow; p, rapid; q, strong; r, weak; s, fine; t, slippery u, rough; $\mathrm{v}$, surging. Blue color and red color denote yes, and no respectively.

excess/deficiency, and the overall summary principles of YinYang. Excess and deficiency indicate the relative strength of the pathogenic factor and the Qi $[13,14]$.

The explanatory variable with the greatest impact on the assessment was a pulse diagnosis variable known as the fine pulse, and the diagnosis was related to both types of deficiencies. The tongue diagnosis variable of pale tongue was also highly relevant as an explanatory factor. Fine pulse and pale tongue are important factors for the diagnosis of the QD pattern according to K-SPI-III [5].

As a result of the second axis quantification, the external criteria were divided into either the DP or non-DP patterns. The DP and non-DP cases can be distinguished by the presence or absence of a pathological product known as DP. The DP patterns, which is classified by etiology, is a combination of phlegm and internal dampness causing disease. Slippery pulse was the explanatory variable with the greatest effect on the division. Kim's study [2] also reported that slippery pulse was the most potent factor for determining the DP pattern.

Comparing the results with the K-SPI-III confirms that the results agree in many aspects with the exception of only few elements. The result shows that specific tongue and pulse indicators are important factors for the difficult diagnosis of excess/deficiency.
Actual diagnoses are performed using the pulse diagnosis and tongue diagnosis methods and by pooling information from the four diagnostic methods. Therefore, future studies should perform these types of analyses by considering pooled information from the four diagnostic methods. Tongue and pulse diagnoses depend on the clinician's experience and knowledge and a variety of environmental factors. It is essential to establish an objective diagnostic standard for tongue and pulse diagnoses such as detailed SOPs or other diagnosis tools.

Although there were some limitations, the results of this study support the objectivity of the theories of TKM. In addition, this study can be considered significant because it attempted to build a model using a statistical method to objectively quantify PI and various indicators that constitute the unique diagnosis system of TKM. Furthermore, the results of this study should be very helpful in the selection, development, and modification of future diagnostic standards in TKM for stroke PI.

\section{Acknowledgment}

This research was supported by a grant from the Korea Institute of Oriental Medicine (K12130). 


\section{References}

[1] T. Deng, Basic Theory of Traditional Chinese Medicine, vol. 9, Shanghai Scientific and Technical Publishers, Shanghai, China, 1984.

[2] H. J. Kim, H. S. Bae, S. U. Park, S. K. Moon, J. M. Park, and W. S. Jung, "Clinical approach to the standardization of oriental medical diagnostic pattern identification in stroke patients," Evidence-Based Complementary and Alternative Medicine, vol. 2011, Article ID 768492, 7 pages, 2011.

[3] S. M. Choi, K. S. Yang, S. H. Choi et al., "Standardization and unification of the terms and conditions used for diagnosis in oriental medicine (III)," Korea Journal of Oriental Medicine, vol. 3, no. 1, pp. 41-65, 1997.

[4] T. Y. Park, J. A. Lee, M. H. Cha et al., "The fundamental study of the standardization and objectification of pattern identification in traditional Korean medicine for stroke (SOPI-Stroke): an overview of phase 1," European Journal of the Integrative Medicine, vol. 4, pp. e125-e131, 2012.

[5] J. A. Lee, J. S. Lee, B. K. Kang et al., "Report on the Korean standard pattern identification for the stroke-III," Korean Journal of Oriental Internal Medicine, vol. 32, no. 2, pp. 232-242, 2011.

[6] J. A. Lee, T. Y. Park, J. S. Lee et al., "Developing indicators of pattern identification in patients with stroke using traditional Korean medicine," BMC Research Notes, vol. 5, article 136, 2012.

[7] C. Hayashi, "On the quantification of qualitative data from the mathematico-statistical point of view-an approach for applying this method to the parole prediction," Annals of the Institute of Statistical Mathematics, vol. 2, no. 1, pp. 35-47, 1950.

[8] Y. Tanaka, "Review of the methods of quantification," Environmental Health Perspectives, vol. 32, pp. 113-123, 1979.

[9] T. Suzuki and A. Kudo, "Recent application of quantification II in Japanese medical research," Environmental Health Perspectives, vol. 32, pp. 131-141, 1979.

[10] Y. K. Shin, "A study on the quantification for Oriental Medicine Data," Journal of Statistical Theory \& Methods, vol. 8, no. 2, pp. 173-181, 1997.

[11] R. H. Jeon, I. S. Lee, K. K. Kim, and C. W. Kang, "Study on the quantification method of symptoms and signs for the oriental gynaecology experiments," Journal of the Korean Data Analysis Socity, vol. 1, no. 1, pp. 53-63, 1999.

[12] M. M. Ko, J. S. Lee, B. K. Kang, D. S. Oh, and O. S. Bang, "A study on the pattern identification diagnosis of the stroke using quantification method type II," Journal of the Korean Data Analysis Socity, vol. 12, no. 4, pp. 1875-1887, 2010.

[13] J. K. Yong, "A view on the standardization process of differentiation system and searched," Journal of Hyungok Academic Society, vol. 1, no. 1, pp. 37-44, 1996.

[14] G. Maciocia and Z. Z. Ying, The Practice of Chinese Medicine: The Treatment of Diseases with Acupuncture and Chinese Herbs, Churchill Livingstone, Edinburgh, UK, 1994. 


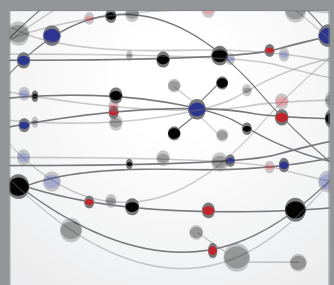

The Scientific World Journal
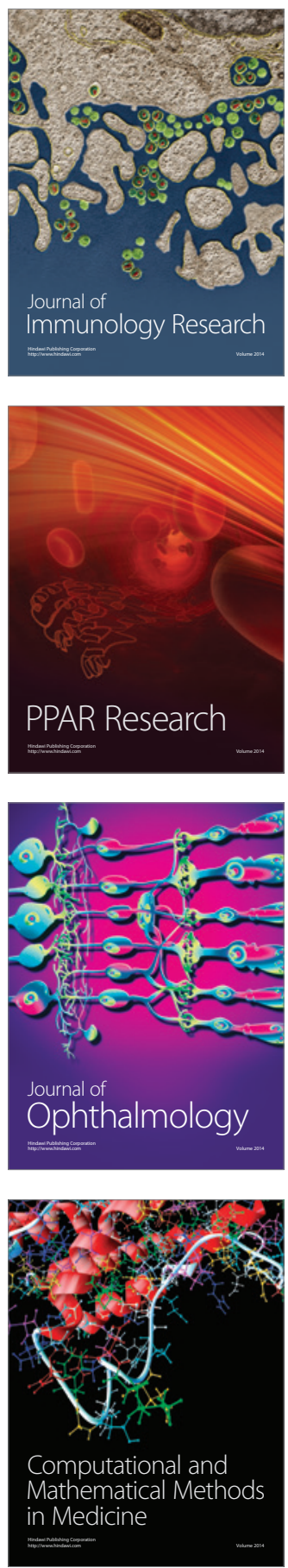

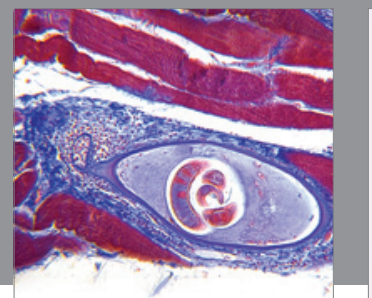

Gastroenterology

Research and Practice
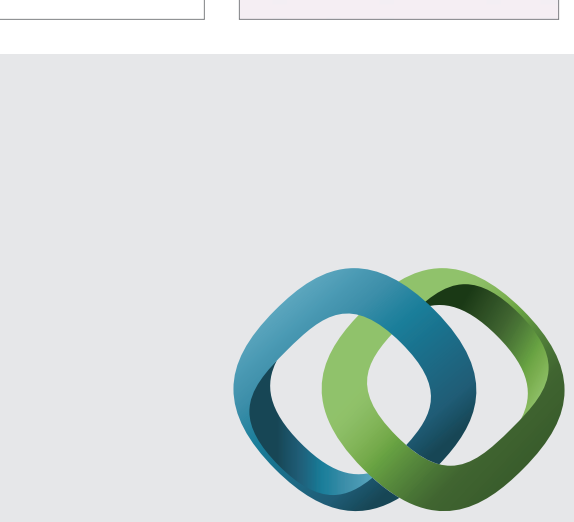

\section{Hindawi}

Submit your manuscripts at

http://www.hindawi.com
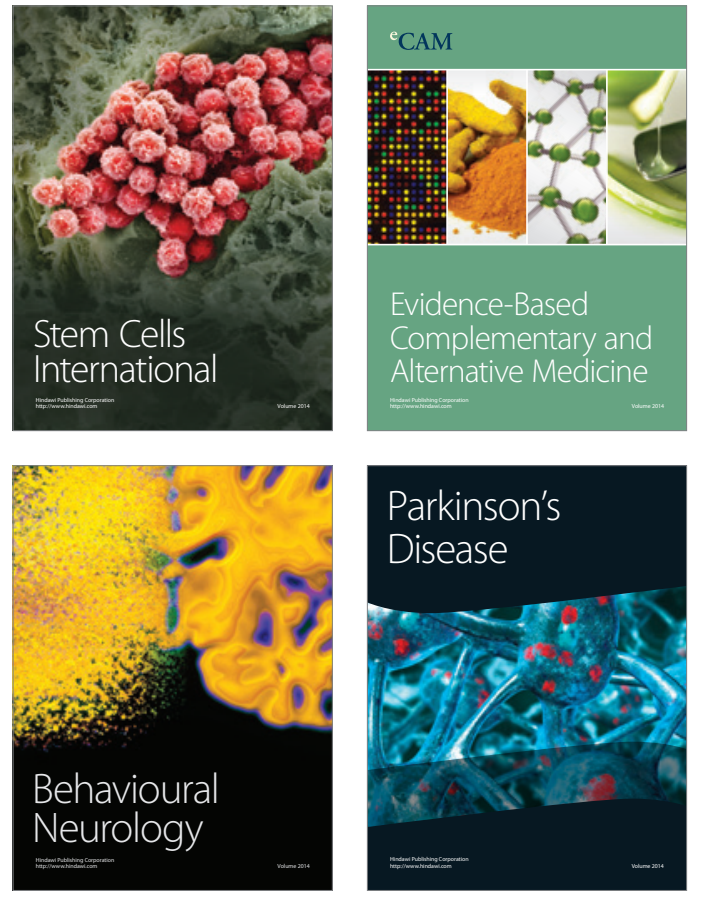
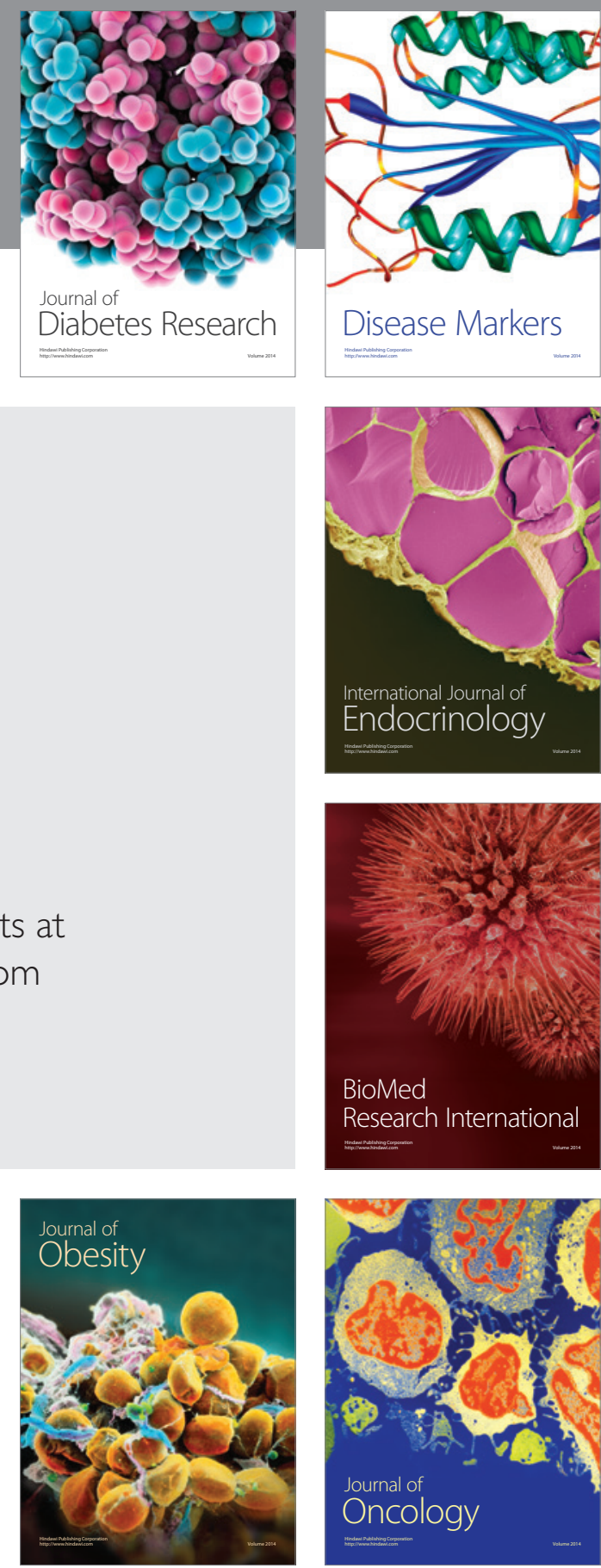

Disease Markers
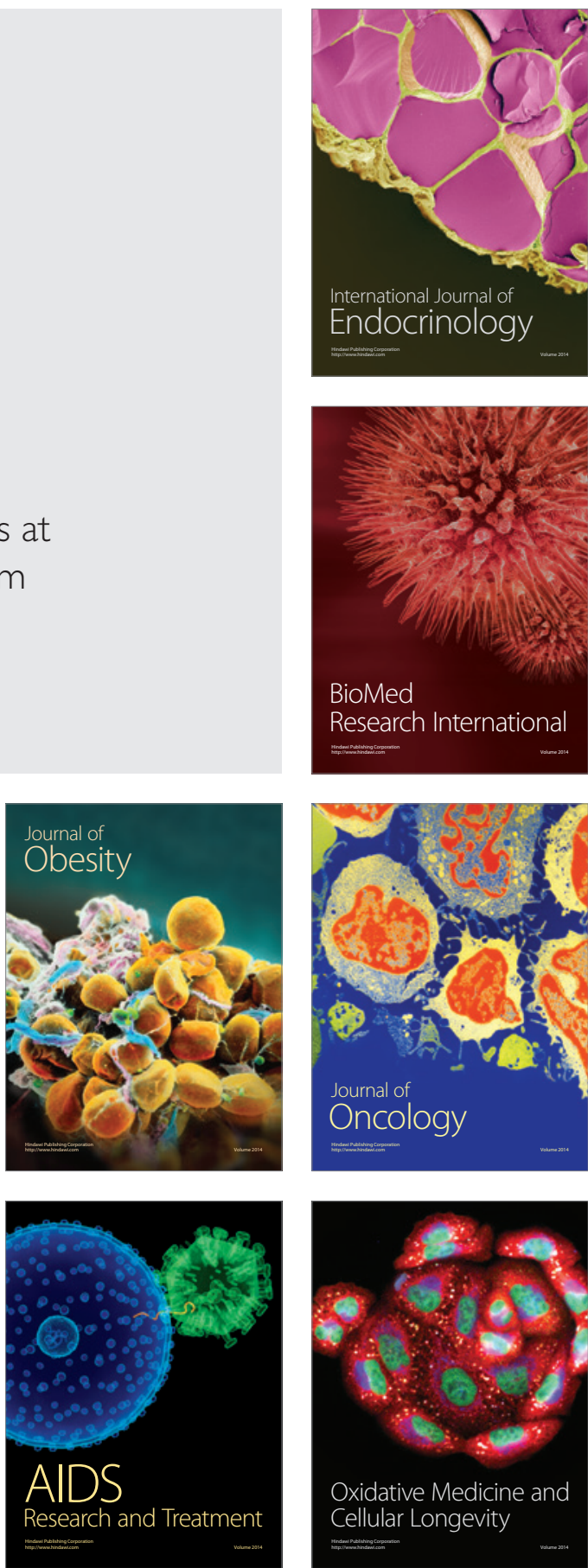